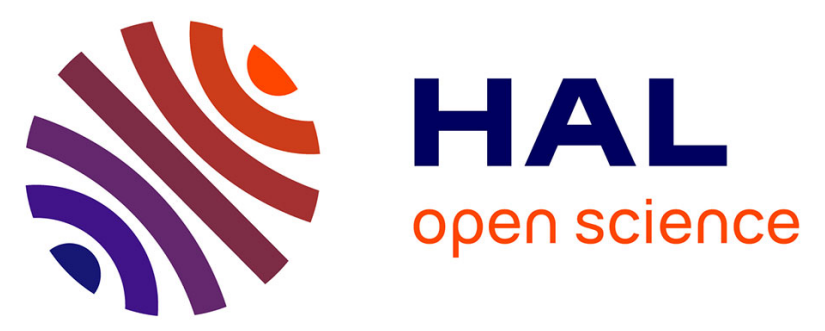

\title{
Mucoadhesive paclitaxel-loaded chitosan-poly (isobutyl cyanoacrylate) core-shell nanocapsules containing copaiba oil designed for oral drug delivery
}

\author{
F.H. Xavier-Jr, C. Gueutin, H. Chacun, Christine Vauthier, E.S.T. Egito
}

\section{To cite this version:}

F.H. Xavier-Jr, C. Gueutin, H. Chacun, Christine Vauthier, E.S.T. Egito. Mucoadhesive paclitaxelloaded chitosan-poly (isobutyl cyanoacrylate) core-shell nanocapsules containing copaiba oil designed for oral drug delivery. Journal of Drug Delivery Science and Technology, 2019, 53, pp.101194. 10.1016/j.jddst.2019.101194 . hal-02983982

\section{HAL Id: hal-02983982 \\ https://hal.science/hal-02983982}

Submitted on 30 Oct 2020

HAL is a multi-disciplinary open access archive for the deposit and dissemination of scientific research documents, whether they are published or not. The documents may come from teaching and research institutions in France or abroad, or from public or private research centers.
L'archive ouverte pluridisciplinaire HAL, est destinée au dépôt et à la diffusion de documents scientifiques de niveau recherche, publiés ou non, émanant des établissements d'enseignement et de recherche français ou étrangers, des laboratoires publics ou privés. 


\title{
Mucoadhesive paclitaxel-loaded chitosan-poly (isobutyl cyanoacrylate) core-shell nanocapsules containing copaiba oil designed for oral drug delivery
}

\author{
Xavier-Jr., F.H. ${ }^{1,2,3}$, Gueutin, C. ${ }^{2}$, Chacun, H. ${ }^{2}$, Vauthier, C. ${ }^{2}$, Egito, E.S.T. ${ }^{*}$
}

1 Universidade Federal do Rio Grande do Norte, Centro de Ciências da Saúde, Departamento de Farmácia, Laboratório de Sistemas Dispersos (LaSiD). Av. Gal. Gustavo Cordeiro de Farias, S/N, Petrópolis, 59010-180, Natal-RN-Brazil.

${ }^{2}$ Université Paris Sud, Institut Galien Paris-Sud - UMR CNRS 8612 - Faculté de Pharmacie, 92296 - Chatenay-Malabry Cedex - France.

${ }^{3}$ Programa de Pós-Graduação em Biotecnologia, Universidade Potiguar (UnP), Campus Salgado Filho, 59075-000, Natal - RN, Brazil. \#Current address

Published in: JDDST 2019; 53:101194. https://doi.org/10.1016/i.jddst.2019.101194

* Corresponding author:

Professor Eryvaldo Sócrates Tabosa do Egito, $\mathrm{PhD}$

Rua Jaguarari, 4985 - Apt. 1603D. Candelaria, Natal - Brazil (59064-500)

Phone: +5584994318816 or +558433429817

E-mail address: socratesegito@gmail.com 


\begin{abstract}
The development of drug delivery systems for potent anticancer drugs like paclitaxel remains a challenger. The aim of this work was to study the mucoadhesive properties of paclitaxelloaded chitosan-poly (isobutyl cyanoacrylate) core-shell nanocapsules designed for oral drug delivery. Using an experimental design approach, the nanocapsules were produced and, then, physicochemically characterized. Mucoadhesion assays were performed in-vitro by the aggregation test with mucin and ex-vivo, in Ussing Chamber, using freshly excised rat intestinal mucosa. [3H]-paclitaxel dosages were carried out by liquid scintillation. Paclitaxel-loaded nanocapsules showed a mean hydrodynamic diameter of $470 \mathrm{~nm}$ with low polydispersity index and spherical form. Encapsulation efficiency and drug loading of paclitaxel were $74 \pm 1 \%$ and $1.70 \pm 0.02 \%$, respectively. After drying, nanocapsules could be redispersed with no changes on their nanostructure. Dispersions of nanocapsules were stable in simulated gastric medium for $120 \mathrm{~min}$, and after six months of storage at $4^{\circ} \mathrm{C}$. They showed interesting mucoadhesive properties with mucins and good association ( $9 \%$ ) with the intestinal mucosa of the rat. Taking together, results from the present work are encouraging to pursue the development of chitosan-coated nanocapsules for oral delivery of paclitaxel as new treatment for cancer with possible synergetic anticancer effect with the therapeutically active components found in copaiba oil.
\end{abstract}

\title{
Graphical Abstract
}

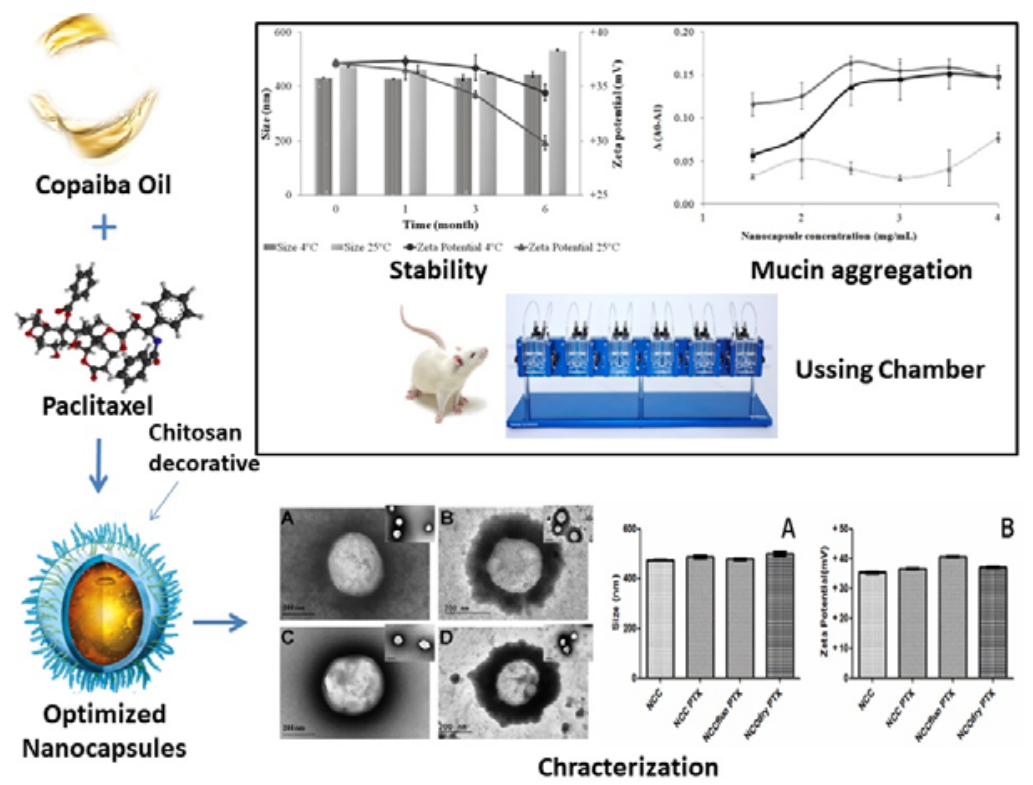

Keywords: Mucoadhesion; Nanocapsules; Paclitaxel; Oral Delivery; Poly(isobutyl cyanoacrylate); Chitosan; Copaiba Oil. 


\section{Introduction}

Cancer remains a major cause of death in most countries in the world, and its incidence increases over the years (Lee et al., 2017). Recently, many works have been focused on the development of oral anticancer drugs to improve the ease of treatments for patients (Mazzaferro et al., 2013; Roger et al., 2010; Wei et al., 2015). Paclitaxel $\left(\mathrm{C}_{47} \mathrm{H}_{51} \mathrm{NO}_{14}\right.$ ) is a pseudoalkaloid anticancer drug with a diterpenoid structure, extracted from the bark of the Pacific yew tree (Taxus brevifolia) (Wani et al., 1971). This drug's mechanism of action is based on the inhibitory effect of cellular growth by hyper-stabilizing the cellular microtubules. Indeed, cell replication in the late G2 mitotic phase of the cell cycle is inhibited, which in turn leads to apoptosis. Paclitaxel has a powerful antitumor ability against a wide spectrum of cancers such as breast and lung cancers, acute leukemia, advanced ovarian, head and neck carcinomas (Rivkin et al., 2010). Theoretically, oral administration of paclitaxel, as for many other drugs, is a preferable choice compared to other routes due to several advantages: higher convenience for patient, hence, better compliance to treatment, lower cost and higher safety (McConville, 2017). Unfortunately, paclitaxel, which is insoluble in aqueous based medium and metabolized over absorption by epithelial cells of the gut mucosa, shows a limited oral bioavailability $(<10 \%)$, which complicates its oral administration (Peltier et al., 2006).

Nowadays, strategies based on the use of nanoparticles are proposed to overcome these limitations. Indeed, it was shown that the association of drugs with nanoparticles may be efficient to increase bioavailability of many drugs, including paclitaxel, by protecting them against degradation and, eventually, enhancing their permeability across the intestinal epithelium (Calleja et al., 2015; Ponchel and Irache, 1998; Wang et al., 2017; Zabaleta et al., 2013). Additionally, nanoparticle systems can reduce toxicity by controlling the drug biodistribution from the blood compartment and, once in the target tissue, enhancing the delivery of the drug to resistant cancer cells over expressing the P-glycoprotein (Agueros et al., 2010; Calleja et al., 2014; Jabr-Milane et al., 2008).

A large part of the published works reported the delivery of drugs, including paclitaxel, after association with nanospheres. Drawbacks of these systems are the, generally, low payload due to the low amount of drug that can compose the nanostructure. Nanocapsules, which are vesicles, appear more suitable systems to achieve high payload, especially when the drug is soluble in the component of the nanocapsules core cavity and have favorable partition coefficient to remain in this medium during the nanocapsules preparation (Buzea et al., 2007).

Poly(isobutyl cyanoacrylate) nanocapsules has been used as a "passive tumor targeting" due to the accumulation in certain solid tumors induced by the enhanced permeability and retention effect (Danhier et al., 2010). Also, such systems are attractive to enhance drug delivery by oral route, as suggested from previous works carried on drug and protein delivery (Anand et al., 2016; Anand et al., 2015; Shamekhi et al., 2018; Thwala et al., 2018). It has been also demonstrated that Poly(isobutyl cyanoacrylate) nanocapsules were stable in gastric environment while they appeared to be rapidly translocated in the blood from the intestine, despite observations of relative in vitro instability in simulated intestinal medium (Aboubakar et al., 2000). In a previous work, our group designed a new poly(isobutyl cyanoacrylate) nanocapsules, decorated with chitosan, filled with a natural oil and having an interesting biological activity including anticancer properties (Xavier-Junior et al., 2018). However, the concept of drug delivery has not been demonstrated.

Therefore, the aim of the present work was to investigate the encapsulation of paclitaxel in the copaiba oil nanocapsules decorated with chitosan and to evaluate their potential to interact with the gut mucosa of rats thanks to the presence of chitosan on their surface. The new 
nanocapsules exhibiting chitosan on their surface are expected to demonstrate mucoadhesive properties, which are assumed to further potentialize the oral administration of the drug. Paclitaxel would be a suitable drug candidate to incorporate in these nanocapsules due to its high solubility in copaiba oil, as recently demonstrated, (Xavier-Junior et al., 2016).

Paclitaxel-loaded nanocapsules were produced by interfacial polymerization of isobutyl cyanoacrylate and optimized by a statistical interaction approach varying copaiba oil, isobutyl cyanoacrylate and paclitaxel concentrations. Stability of paclitaxel-loaded nanocapsules were, then, evaluated in simulated gastrointestinal fluids, under different storage conditions and after drying. Mucoadhesive properties were evaluated based on an aggregation test with mucins and on the evaluation of their retention at the level of rat intestinal mucosa mounted in an Ussing Chamber.

\section{Materials and Methods}

\subsection{Materials}

Isobutyl cyanoacrylate was provided by ORAPI engineered solutions worldwide (Vaulx-enVelin, France). Copaiba oil was purchased from Flores \& Ervas (Piracicaba, SP, Brazil). Chitosan 20,000 Da was purchased from Amicogen (Jinju, South Gyeongsang, South Korea). PolyFluor ${ }^{\circ}$ 570: methacryloxyethyl thiocarbamoyl rhodamine B (N-[9-(2-carboxy-x-methacryloxyethylthiocarbamoylphenyl)-6-diethylamino-3H-xanthen-3-ylidene]-N-ethyl-ethanaminium chloride) was provide from Biovalley Polyscience (Marne-la-Vallée, France). Paclitaxel was obtained from CHEMOS GmbH (Regenstauf, Germany). [3H]-paclitaxel (3 Ci/mmol) was purchased from Isobio (Fleurus, Belgium). Hionic-Fluor ${ }^{\circ}$ and Ultima-Gold ${ }^{\circ}$ (Packard, Rungis, France) were used as scintillating cocktails for radioactive analyses. Soluene- $350^{\circ}$, used to dissolve biological samples, was obtained from Perkinelmer (Courtaboeuf, France). Pancreatin, pepsine, sodium chloride, hydrochloric acid, sodium hydroxide, monobasic potassium phosphate and mucin, from porcine stomach, were purchased from Sigma-Aldrich (SaintQuentin Fallavier, France). Ethanol, acetonitrile and nitric acid were provided by Fisher Scientific (IIlkirch, France). Ultrapure water was obtained from a Millipore purification system (Milli-Q plus, Millipore, St Quentin en Yvelines, France). All chemicals were of reagent grade and used as received.

\subsection{Preparation of nanocapsules}

Nanocapsules were prepared by interfacial polymerization as described by Al Khouri Fallouh et al (Al Khouri Fallouh et al., 1986) following the modification introduced by Xavier-Jr. et al.(Xavier-Junior et al., 2018). Briefly, an organic phase composed of ethanol (6.25 mL), copaiba oil $(0.250$ and $0.450 \mathrm{~mL})$, isobutyl cyanoacrylate $(0.032$ and $0.042 \mathrm{~mL})$ and paclitaxel ( 2 and 10 $\mathrm{mg}$ ) was introduced dropwise in an $5{ }^{\circ} \mathrm{C}$ aqueous medium containing $12.5 \mathrm{~mL}$ of chitosan $(0.9$ $\%)$, which $\mathrm{pH}$ was adjusted at 3 . Stirring rate was fixed at 1,250 rpm during the addition and over the next 10 minutes. Ethanol was, then, evaporated using a rotary evaporator at $35^{\circ} \mathrm{C}$ for $20 \mathrm{~min}$ at $43 \mathrm{mBa}$ (BÜCHI Rotavapor R-125, Heating Bath B-491, Vacuum pump V-700, recirculating Chiller F-108, Flawil, Switzerland). The obtained nanocapsule dispersions were filtered ( $5 \mu \mathrm{m}$ nylon membrane filter, Merck Millipore, Billerica, MA, EUA). Then, they were purified and concentrated to $2 \mathrm{~mL}$ in an Amicon Ultra centrifugal filter, $100 \mathrm{kDa}$ molecular weight cut off (Merck Millipore, Billerica, MA, USA). This system was placed in centrifuge (Eppendorf centrifuge $5804 \mathrm{R}$, Rotor S-4-72, Hamburg, Germany) under 4,000 g at $20{ }^{\circ} \mathrm{C}$ against Milli- $Q^{\circ}$ water three times for 20 min. Optimization of the paclitaxel encapsulation was achieved using a statistical interaction approach among the concentrations of copaiba oil, 
isobutyl cyanoacrylate and paclitaxel variables. Particle size and zeta potential were the dependent variables.

For the mucoadhesion and stability studies, nanocapsules were labeled with PolyFluor 570 : methacryloxyethyl thiocarbamoyl rhodamine $\mathrm{B}$, and with [3H]-paclitaxel. The nanocapsules were prepared following the protocol described above with minor changes. Initially, $0.25 \mu \mathrm{L}$ of PolyFluor 570 (1 mg. $\mathrm{mL}^{-1}$ in ethanol) was added in the organic phase. Immediately, in this phase, copaiba oil and isobutyl cyanoacrylate were added, to produce fluorescent nanocapsules. For radiolabeled nanocapsules containing paclitaxel, $4.2 \mathrm{kBq}$ of [3H]-paclitaxel per $\mathrm{mL}$ of final nanocapsules suspension was dissolved in organic phase before polymerization. For some experiments non chitosan-coated nanocapsules were also needed. These nanocapsules were prepared with pluronic $\mathrm{F}-68^{\circ}$, according to previous work (Aboubakar et al., 1999).

\subsection{Characterization of nanocapsules}

The hydrodynamic diameter and the size distribution of the nanocapsules were determined by DLS using a Zetasizer Nano ZS9O (Malvern Instruments Ltd, Worcestershire, UK) at $25^{\circ} \mathrm{C}$. The scattered angle was fixed at $90^{\circ}$. Samples were diluted at 1:100 with Milli- $Q^{\circ}$ water before analysis. Results were expressed as the mean hydrodynamic diameter, the standard deviation of the size distribution and the Pdl. Zeta potential of the nanocapsules was measured by Laser Doppler Electrophoresis using a Zetasizer Nano ZS90 (Malvern Instruments Ltd, Worcestershire, UK). To maintain a constant ionic strength, samples were diluted (1:100) in saline solution $(\mathrm{NaCl})$ at $1 \mathrm{mM}$. All results corresponded to the average of three determinations.

TEM was used to investigate the nanocapsules morphology. Observations were performed using a JEOL 1400 transmission electron microscope (JEOL Ltd, Tokyo, Japan) coupled with a Gatan CCD high-resolution digital camera (Orius SC1000). One drop of the diluted nanocapsules in Milli- $Q^{\circ}$ water (1:100) was placed on a formvar-carbon coated copper grid for 5 minutes. Thereafter, unfixed nanocapsules were removed by filter paper and one drop of $2 \%$ phosphotungstic acid ( $\mathrm{pH} 7.4$ ) was added to the grid for 30 seconds. The excess was wiped off by filter paper. Finally, the grid was air dried prior to its introduction in the electron microscope.

Fluorescence microscopy studies were conducted to test the stability of the nanocapsules in simulated gastrointestinal fluids. They were performed using a fluorescent microscope (Leitz Diaplan, Wild Leitz $\mathrm{GmBH}$, Wetzlar, Germany) equipped with a filter N 2.1 adapted to PolyFluor 570: methacryloxyethyl thiocarbamoyl rhodamine B (excitation 515-561 nm, emission cutoff $580 \mathrm{~nm}$ ). The images were captured using QED capture version 2.0.24. A control experiment was performed by diluting the nanocapsules in Milli- $Q^{\circ}$ water $(1: 50)$ instead of using simulated gastrointestinal fluids. The same parameters were maintained for all samples.

\subsection{Determination of paclitaxel}

\subsubsection{HPLC analysis}

The amount of paclitaxel associated to the nanocapsules was quantified by high-performance liquid chromatography (HPLC). The method was developed and validated in a previous work (Xavier-Junior et al., 2016). The used chromatographic system was a Waters 515 pump, a Waters 717 plus autosampler and a Waters 486- Tunable Absorbance detector (Waters Corp., 
Milford, MA). Chromatographic separations were achieved using a Uptisphere Strategy 100A reversed-phase C-18 (150 mm x $3 \mu \mathrm{m} \times 3 \mathrm{~mm}$ ) column and a Uptisphere Strategy C18-2 guard column (10 mm x $3 \mu \mathrm{m} \times 4 \mathrm{~mm}$ ) (Interchim SA, France). The mobile phase, pumped at 0.4 $\mathrm{mL} . \mathrm{min}^{-1}$, was acetonitrile:water (50:50) at $30{ }^{\circ} \mathrm{C}$ monitored with UV-detection at $228 \mathrm{~nm}$. The mobile phase and the samples were filtered through a $0.20 \mu \mathrm{m}$ hydrophilic nylon membrane filter (Merck Millipore, Billerica, MA, EUA) prior to use. Under these conditions, the run time was $15 \mathrm{~min}$ and the paclitaxel was eluted at a retention time of $9.7 \mathrm{~min}$. Chromatographic data were monitored and analyzed using UV Winilab3 software (Perkin Elmer, Shelton, USA). The method was validated demonstrating that it was linear $\left(r^{2}=0.999\right)$ within the range of concentration comprised between 200 to $1700 \mathrm{ng} \cdot \mathrm{mL}^{-1}$. The recovery ranged from 99.0 to $103.2 \%$ and the relative standard deviation for intra- and inter-day precision was less or equal to $2.03 \%$. The specificity was tested in presence of the nanocapsules' adjuvants and demonstrated that these factors did not alter the paclitaxel assay. The limit of quantification and limit of detection were 33.8 and $10.1 \mathrm{ng} \cdot \mathrm{mL}^{-1}$, respectively.

\subsubsection{Radioactivity analysis}

Radiolabeled [3H]-paclitaxel loaded copaiba oil- poly(isobutyl cyanoacrylate) nanocapsules coated with chitosan was determined by liquid scintillation counter (Model LS 6000 TA, Beckman, France). Samples $(40 \mu \mathrm{L})$ were vortexed for 1 minute with $10 \mathrm{~mL}$ of a scintillating cocktail and analyzed. For analysis in the rat intestinal tissue, $1 \mathrm{~cm}^{2}$ from excised tissue was digested in $2 \mathrm{~mL}$ of Soluene- $350^{\circ}$ at $65{ }^{\circ} \mathrm{C}$ overnight. Then, $10 \mathrm{~mL}$ of scintillating cocktail was added into the bottle, vortexed for 1 minute and the [3H]-paclitaxel radioactivity was measured.

\subsection{Drug loading and encapsulation efficiency}

Free drug was determined in the clear supernatant obtained after separation of nanocapsules from aqueous dispersion medium by the ultrafiltration-centrifugation technique (Microcon centrifugal filter, Ultracel YM-100, regenerated cellulose, Merck Millipore, Billerica, MA, USA). Nanocapsules were centrifuged at 10,000 rpm for 20 min (Eppendorf centrifuge 5418, Rotor FA-45-18-11, Hamburg, Germany) over the ultrafiltration unit. Drug loading (DL) and encapsulation efficiency (EE) were expressed as percentages and deduced from equations 1 and 2, respectively:

DL $(\%)=\frac{\text { Weight. of nannasanules las ded paclitaxel }}{\text { Total weight of nanocapsules }} \times 100 \%$ (1)

EE $(\%)=\frac{\text { Fixperimental drug lnading }}{\text { Theorical drug, loading }} \times 100 \%(\mathbf{2})$

\subsection{Stability of paclitaxel loaded nanocapsules}

The stability of paclitaxel loaded copaiba oil- poly(isobutyl cyanoacrylate) nanocapsules coated with chitosan dispersion was evaluated for over a period of 6 months in terms of size and zeta potential, while storage stability was achieved at $4{ }^{\circ} \mathrm{C}$ and $25^{\circ} \mathrm{C}$. Stability studies of dried nanocapsules dispersion were investigated considering possible physicochemical instability and also future application as oral tablets containing these nanosystem. Thus, nanocapsules 
dispersion was dried in Eppendorf Vacufuge 5301 vacuum centrifuge (Eppendorf AG, Hamburg, Germany) at room temperature. The dried nanocapsules were analyzed by DLS and TEM after rehydration and redispersion in the equivalent amount to recovered initial volume of Milli-Q water.

Stability of the nanocapsules was also investigated after incubation in simulated gastrointestinal fluids according to the conditions described in the United States Pharmacopoeia XXXIV (Convention, 2011), in order to predict the effect of gastrointestinal media under nanocapsules after oral administration. Simulated gastric fluid medium was composed by $0.2 \%$ of sodium chloride, $8 \%$ of hydrochloric acid $(1 \mathrm{M})$ and $0.32 \%(\mathrm{w} / \mathrm{v})$ of pepsin with a $\mathrm{pH}$ of 1.2. Simulated intestinal fluid medium was made of $0.62 \%$ monobasic potassium phosphate solution and $7.7 \%$ of sodium hydroxide with pancreatin $1 \%(\mathrm{w} / \mathrm{v})(\mathrm{pH}$ 6.8). For this study, nanocapsules labeled with PolyFluor 570 were added in the simulated fluid at dilution of $1: 50$ and incubated at $37^{\circ} \mathrm{C}$ for 120 minutes. At defined times, the samples were collected and analyzed by DLS for size measurement. Integrity of the nanocapsules was appreciated by fluorescence microscopy observations as previously described.

\subsection{Evaluation of the mucoadhesion}

\subsubsection{Aggregation of nanocapsules in presence of mucin}

Mucin from porcine stomach at $1 \%(\mathrm{w} / \mathrm{v})$ was dispersed in deionized water for $2 \mathrm{~h}$ at room temperature. Nanocapsules were used at different concentrations: 1.5, 2.0, 2.5, 3.0, 3.5 and $4.0 \mathrm{mg} \cdot \mathrm{mL}^{-1}$. Then, $75 \mu \mathrm{L}$ of the nanocapsules dispersion was placed in a 96 - microwell polystyrene conical bottom plate. The initial absorbance $\left(A_{0}\right)$ of each dispersion was evaluated at $450 \mathrm{~nm}$ using a microplate reader (Multiskan Anscent, Labsystems SA, Cergy-Pontoise, France). $30 \mu \mathrm{L}$ of mucin suspension $\left(0.25 \mathrm{mg} \cdot \mathrm{mL}^{-1}\right)$ was added and the plate was incubated for $1 \mathrm{~h}$ at $37{ }^{\circ} \mathrm{C}$. To quantify the aggregation, the absorbance $A_{1 \mathrm{~h}}$ was evaluated after centrifugation ( $240 \mathrm{~g}$ ) for $5 \mathrm{~min}$ at $25{ }^{\circ} \mathrm{C}$ (Eppendorf centrifuge $5804 \mathrm{R}$, Rotor A- 4- 81 with MTP/Flex carrier, Hamburg, Germany). Each experiment was repeated three times and the difference between $A_{0}$ and $A_{1 h}\left(A_{0}-A_{1 h}\right)$ was plotted as a function of the dilution performed for each nanocapsules dispersion.

\subsubsection{Mucoadhesion assay on rat intestinal mucosa}

The mucoadhesion of the nanoparticles under rat intestinal mucosa was studied by Ussing Chamber. Animal experiments were carried out according to the recommendations of the ethics committee of the French Ministry of Higher Education and Research, project 2003-055 regarding the care and use of animals for experimental procedures. Male Wistar rats (200-250 g) (Charles River, Paris) were used for the mucoadhesion ex vivo assays. Rats were allowed free access to water and food, and housed under controlled environmental conditions (constant temperature, humidity, and a $12 \mathrm{~h}$ dark-light cycle). Animals were euthanized with an overdose of pentobarbital by intraperitoneal injection. Fresh small intestine (jejunum) portion was excised, rinsed with physiological saline $(\mathrm{NaCl} 0.9 \%)$ and cut into small segments of 2-3 $\mathrm{cm}$ length. After visual examination of the tissue, sections containing Peyer's patches were discarded.

Intestinal portions were mounted in Ussing Chambers with a delimited intestinal mucosa surface area $\left(1 \mathrm{~cm}^{2}\right)$. The systems were maintained in Ringer buffer at $37^{\circ} \mathrm{C}$, continuously oxygenated with $\mathrm{O}_{2} / \mathrm{CO}_{2} 95 \% / 5 \%$. After removing the transport buffer, $0.1 \mathrm{~mL}$ of radiolabeled nanocapsules in Ringer buffer ( $\mathrm{pH}$ 7.5) were applied to the mucosal surface. Each compartment of the Ussing Chamber was filled with $3 \mathrm{~mL}$ of Ringer solution. The experiment 
was performed over a period of 2 hours to ensure the attachment equilibrium. After incubation for 2 hours, the nanocapsules dispersion was removed. Tissue was rinsed three times with $3 \mathrm{~mL}$ of Ringer buffer, to eliminate non-attached nanocapsules, while it was still mounted in the Ussing Chamber. Subsequently, the mucosa with the attached nanocapsules was recovered and let to dissolve in $2 \mathrm{~mL}$ of Soluene- $350^{\circ}$ at $65^{\circ} \mathrm{C}$, overnight. Then, $10 \mathrm{~mL}$ of scintillating liquid was added and, finally, samples were analyzed by liquid scintillation to determine the amount of $[3 \mathrm{H}]$-paclitaxel associated to the mucosa. Results were expressed as the amount of attached nanocapsules per apparent surface $\left(\mathrm{g} / \mathrm{m}^{2}\right)$ and by the number of attached nanocapsules in the tissue, as defined by the Equation 3:

$\mathrm{N}=\frac{\mathrm{m}_{\mathrm{T}} 6}{\rho \mathrm{S} \mathrm{d}}$

where $\mathbf{N}$ is the number of attached nanocapsules, $\mathbf{m}_{\mathbf{T}}$ is the mass of attached nanocapsules $(\mathrm{g})$, $\boldsymbol{\rho}$ is the oil estimated nanocapsules density $\left(0.99 \mathrm{~g} / \mathrm{cm}^{3}\right), \mathbf{d}$ is the nanocapsules diameter $(\mathrm{cm})$ and $S$ is the nanocapsules surface: $S=4 \pi(d / 2)^{2}$. Each sample was tested in three different rats in duplicate.

\subsection{Statistical analysis}

All experiments were conducted in triplicates. All values were expressed as their mean \pm standard deviation (SD). Means of two groups were compared using non-paired Student's ttest. When comparing multiple groups, one way analysis of variance (ANOVA) was applied with the Tukey multiple comparison procedure. The analyses were performed using the Graph Pad Prism (Version 5.0, La Jolla, CA, USA) and the Statistic software (Version 7.0, StatSoft Inc., USA). The statistical data were considered significant at $p<0.05$.

\section{Results and Discussion}

\subsection{Optimization of the preparation of paclitaxel loaded nanocapsules}

Paclitaxel loaded copaiba oil-poly(isobutyl cyanoacrylate) nanocapsules coated with chitosan were produced by the new method of interfacial polymerization of isobutyl cyanoacrylate carried out in the presence of chitosan and without surfactant in the polymerization medium (Xavier-Junior et al., 2018). This method was used to produce chitosan-coated nanocapsules encapsulating paclitaxel assuming that they will show mucoadhesive properties.

Optimization of the incorporation of paclitaxel was studied considering the influence of the isobutyl cyanoacrylate, copaiba oil and paclitaxel concentrations on the size and zeta potential of the produced nanocapsules (Figure 1). It was assumed that nanocapsules with smaller sizes promote mucoadhesion (Bertholon et al., 2006; Ponchel and Irache, 1998). Furthermore, after reaching the blood, small nanocapsules can undergo capillary distribution and uniform perfusion. 

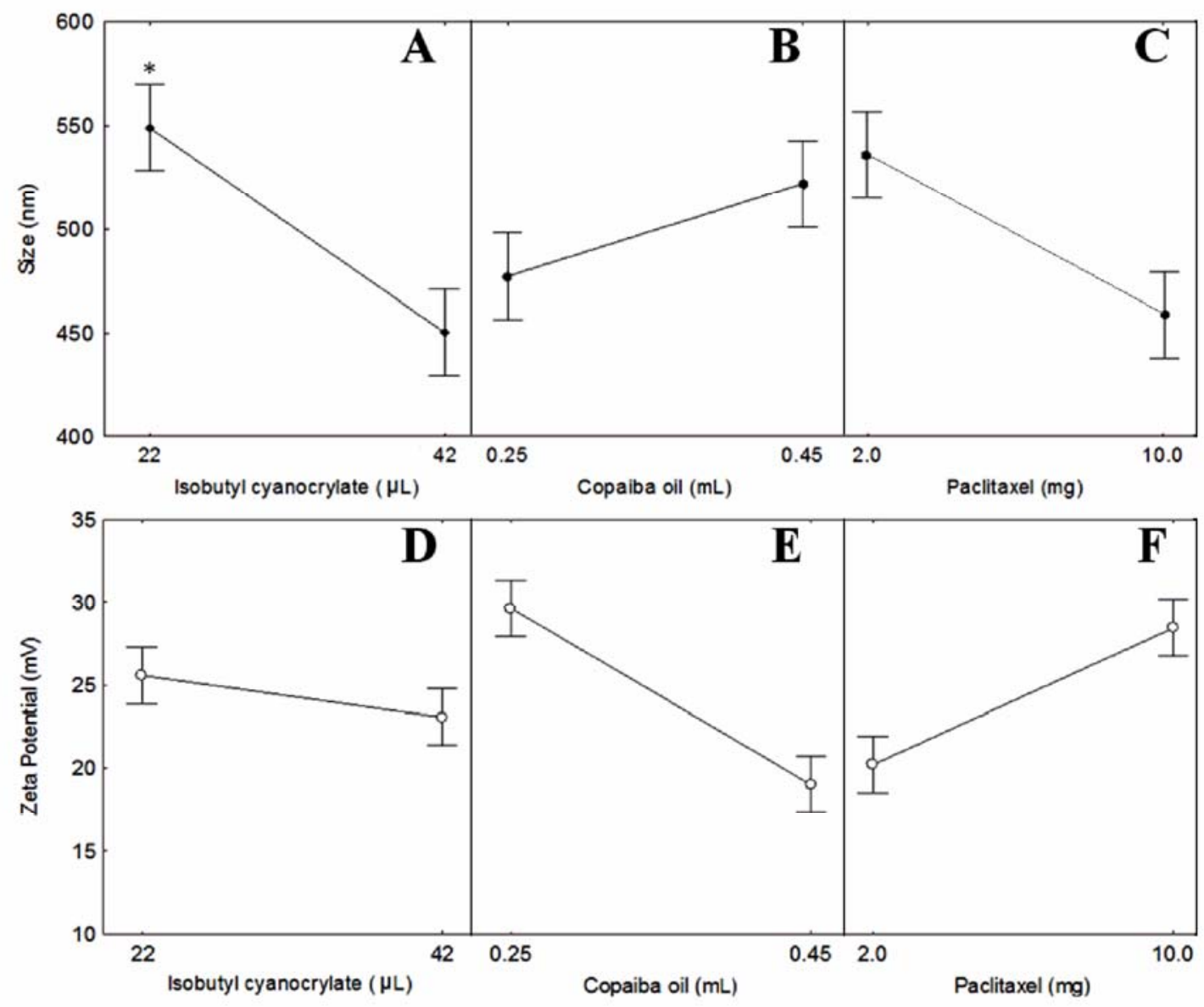

Figure 1- Size (top line) and zeta potential (bass line) influence on paclitaxel loaded nanocapsules formed at low and high concentration of isobutyl cyanoacrylate ( $A$ and $D)$, copaiba oil (B and E) and paclitaxel ( $C$ and F). * Broad distribution size $(n=3)$.

An opposite and significant effect on droplet size was observed when increasing concentrations of isobutyl cyanoacrylate and paclitaxel were added in the polymerization medium $(p<0.05)$. Considering the concentration of the monomer, a decrease on the diameter of the nanocapsules in order of $100 \mathrm{~nm}$ was observed by increasing the amount of isobutyl cyanoacrylate introduced in the polymerization medium from 0.022 to $0.042 \mathrm{~mL}$ (Figure 1A). The nanocapsules dispersion obtained with a concentration of isobutyl cyanoacrylate at the order of $0.022 \mathrm{~mL}$ was unstable, showing not only aggregates, but also a broad size distribution. This can be associated to the low polymer concentration, possibly insufficient to effectively cover the available surface area of the copaiba oil droplets, which were formed during the dispersion of the organic phase in the polymerization medium. By increasing the concentration of the drug from 2 to $10 \mathrm{mg} \cdot \mathrm{mL}^{-1}$ in the organic phase, the diameter of the produced nanocapsules was decreased (Figure 1C). This can be explained by the chemical structure of paclitaxel, in which a slight amphiphilicity can be highlighted and may be enough to promote the formation of emulsion droplets of lower diameter size. In fact, in the absence of paclitaxel this phenomenon was not observed. On the other hand, a slight increase in the nanocapsule diameter size was observed when the volume of copaiba oil entrapped in the systems was increased from 0.25 to $0.45 \mathrm{~mL}$. However, this difference was not statistically significant $(p>0.05)$ (Figure 1B).

A positive zeta potential value was observed to all nanocapsules, suggesting that chitosan was covering the nanocapsules surface as expected. The rather strong positive zeta potential may be explained by the presence of the quaternary ammonium groups in the chitosan (Bathool et 
al., 2012) at the almost neutral $\mathrm{pH}$ of measurement. The positive zeta potential of the nanocapsules was expected to promote mucoadhesion thanks to the electrostatic interactions with the mucus surface, which is negatively charged at physiological pH (Mendes et al., 2018). Higher values of zeta potential (positive or negative) are also highly favorable to obtain stable dispersions of nanocapsules as electrostatic repulsion forces between the particles with the same electric charge prevent the aggregation of the dispersion (Cho et al., 2014). No significant difference $(p>0.05)$ on the zeta potential values was observed for the nanocapsules prepared at low or high concentration of isobutyl cyanoacrylate (Figure 1D). However, the zeta potential value was influenced by the presence and amount of copaiba oil and paclitaxel in the polymerization media. Indeed, copaiba oil reduces the potential zeta value of the formulation, while paclitaxel increases it. (Figure $1 \mathrm{E}$ and F). These phenomena can be explained by the presence of small amounts of copaiba oil and paclitaxel adsorbed at the nanocapsules surface. Also, both molecules can influence the coverage of the nanocapsules surface by the chitosan molecules, leading to masking or exhibiting the positive charge of the polysaccharide (Mohammadpour Dounighi et al., 2012).

In order to obtain nanocapsules of small size, but at the same time with large amount of chitosan coated on their surface, the suggested optimized paclitaxel loaded nanocapsules could be produced using $0.032 \mathrm{~mL}$ of isobutyl cyanoacrylate, $0.25 \mathrm{~mL}$ of copaiba oil and $6 \mathrm{mg}$ of paclitaxel.

\subsection{Characterization of the optimized paclitaxel loaded nanocapsules}

The mean particle diameter of the optimized paclitaxel loaded nanocapsules was found to be $486 \pm 3 \mathrm{~nm}$ with a narrow polydispersity index (Pdl) of 0.17 (Figure 2A). The zeta potential of those nanocapsules was highly positive $(+37.1 \pm 0.3 \mathrm{mV}$ ) (Figure $2 \mathrm{~B}$ ), indicating the presence of chitosan on the nanocapsules surface. Unloaded nanocapsules displayed similar physicochemical properties (particle size, zeta potential and morphology aspects).
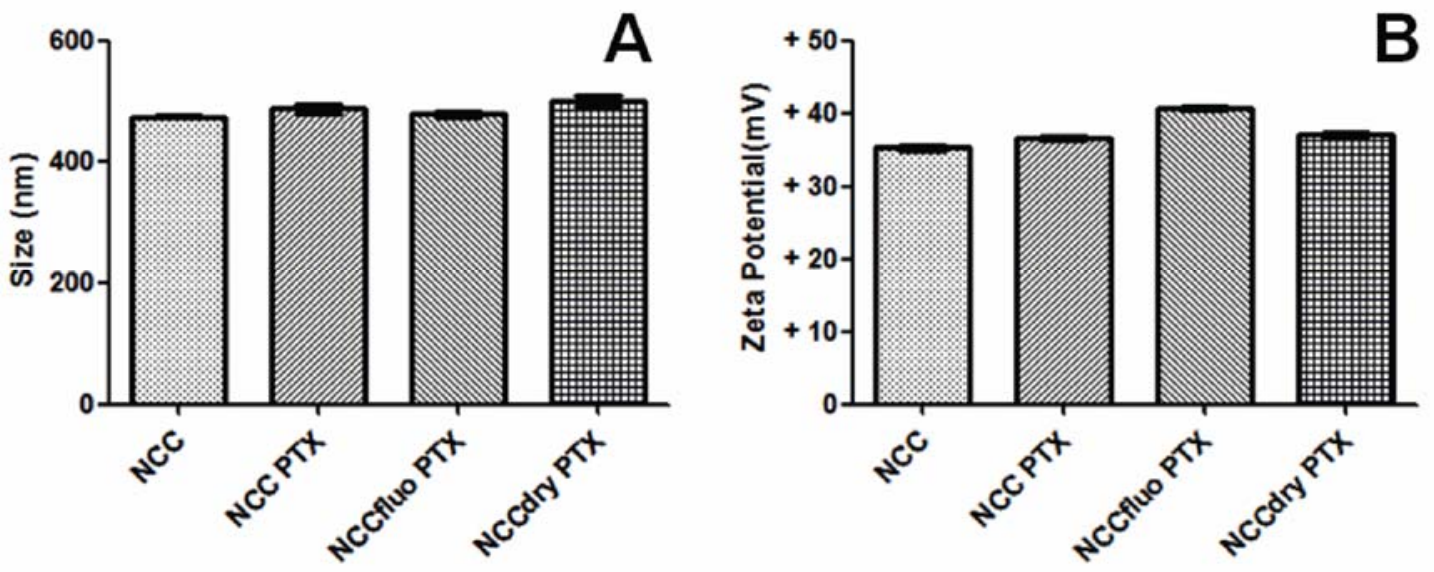

Figure 2: Particle size (A) and zeta potential (B) of different nanocapsules. NCC: Copaiba oilloaded chitosan-poly (isobutyl cyanoacrylate) core-shell nanocapsules; NCC PTX: Paclitaxel loaded NCC; NCCfluo Paclitaxel: PolyFluor 570 labeled NCC PTX; NCCdry PTX: NCC PTX after drying process. $(n=3)$. 
Transmission electron microscopy (TEM) images showed the spherical shape and the surface morphology of the paclitaxel unloaded and loaded nanocapsules (Figure 3 A and B, respectively). The nanocapsules appeared well separated on all preparations. As confirmed by the TEM pictures, nanocapsules were formed with an oily cavity (copaiba oil, in which the paclitaxel was dissolved) surrounded by a polymer membrane. The observed size diameter by microscopy was around $475 \mathrm{~nm}$, which was consistent with values determined by the dynamic light scattering (DLS) measurements.
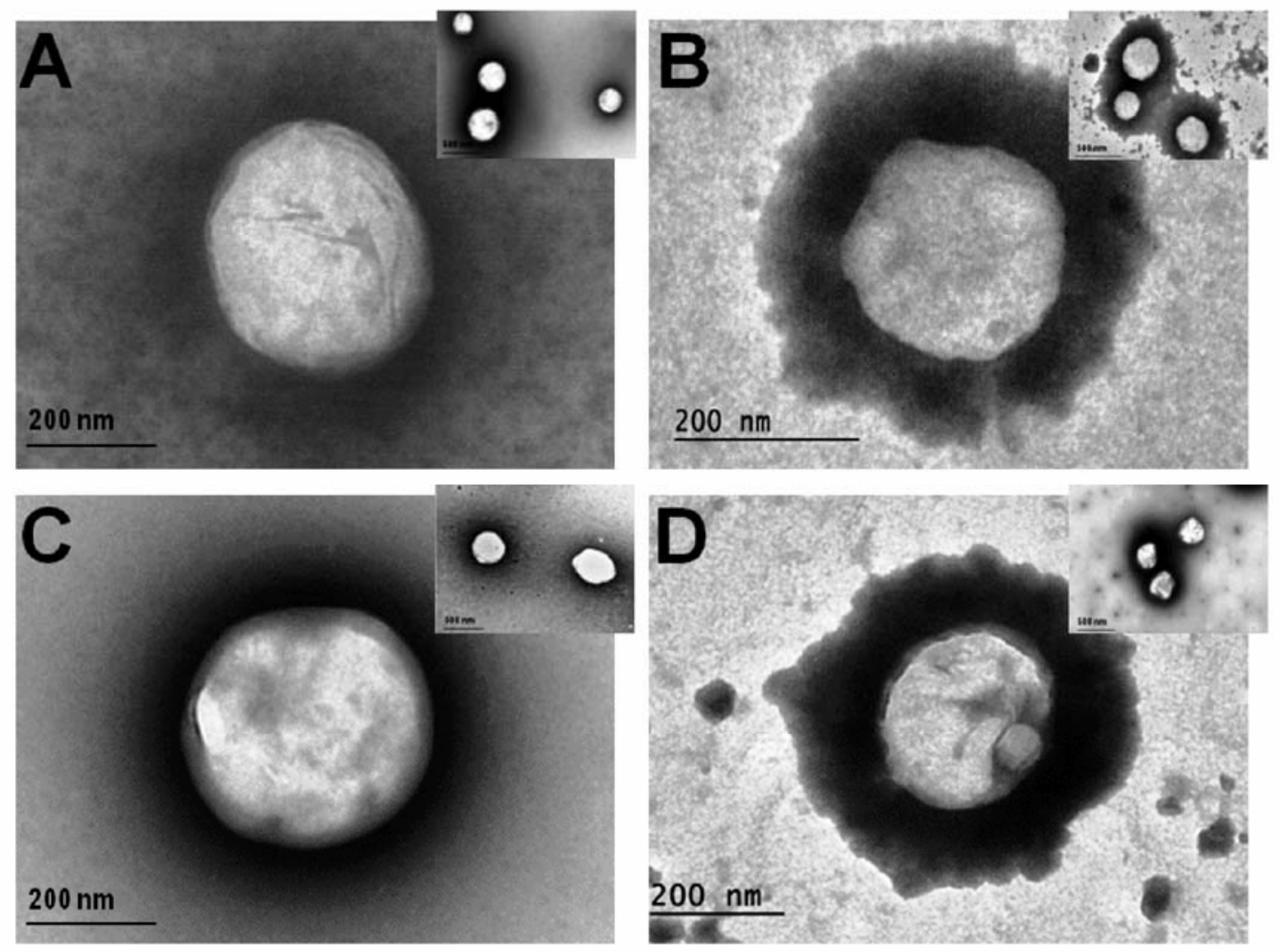

Figure 3- TEM of copaiba oil-loaded chitosan-poly (isobutyl cyanoacrylate) core-shell nanocapsules (NCC) (A); Paclitaxel loaded NCC (NCC PTX) (B); NCC PTX after drying process (C); PolyFluor 570 labeled NCC PTX (D). Scale bar of $200 \mathrm{~nm}$ and $500 \mathrm{~nm}$ to isolated and grouped nanocapsules, respectively.

Concerning the maximum encapsulation efficiency (EE) and drug loading (DL) of paclitaxel in the nanocapsules, the results showed values of $74.5 \pm 1.2 \%$ and $1.7 \pm 0.02 \%(\mathrm{w} / \mathrm{w})$, respectively. The drug concentration loaded in the formulation was $16.8 \pm 0.3 \mu \mathrm{g}$ of paclitaxel per $\mathrm{mg}$ of nanocapsules, corresponding to $746 \pm 12 \mu \mathrm{g}$ of paclitaxel per $\mathrm{mL}$ of nanocapsules dispersion. This result revealed an excellent association between the drug and the nanosystem compared to previously described systems. The DL showed by these nanocapsules was also relevant because was similar to the dose existing for other injectable therapies. For instance, in the Abraxane ${ }^{\circ}$, an albumin bound paclitaxel dosage form, the drug is therapeutically administrated at the concentration of $5 \mathrm{mg} \cdot \mathrm{mL}^{-1}$ of suspension. Previous studies reported an EE of $70 \%$ of paclitaxel into PEGylated poly (lactide-co-glycolide) nanoparticles produced by the nanoprecipitation method (Danhier et al., 2009). Huang et al showed a DL of $0.18 \%$ and $0.56 \%$ for paclitaxel-loaded poly (butylcyanoacrylate) nanoparticle obtained by the emulsion and the microemulsion polymerization methods, respectively (Huang et al., 2007). The high value of DL obtained in this work can be explained by the good solubility of the paclitaxel in the copaiba oil combined to its high partition coefficient in favor to copaiba oil. These features 
favorably contributed to the success of the encapsulation method developed in the present study (Xavier-Junior et al., 2016).

Biopharmaceutical studies require the labeling of particles in order to localize them in vivo and/or in vitro during assays carried out under various experimental conditions (Bravo-Osuna et al., 2007a; Vauthier and Bouchemal, 2009). Fluorescent labeled nanocapsules were synthesized by incorporation of methacryloxyethyl thiocarbamoyl rhodamine B (Polyfluor ${ }^{\circ}$ 570), a fluorescent co-monomer, which reacts with isobutyl cyanoacrylate during the formation of the nanocapsules. Fluorescent labeled nanocapsules did not show significant difference in size and morphology compared to the non-labeled ones. In fact, they only differed by a slight increase of the zeta potential (Figure 2 and 3D). The nanocapsules appeared clearly fluorescent under observation by fluorescent microscopy.

Radiolabeled nanocapsules were effectively developed with $[3 \mathrm{H}]$-paclitaxel. This dispersion showed a radioactive activity of $3.14 \pm 0.07 \mathrm{kbq} \cdot \mathrm{mL}^{-1}$. The encapsulation efficiency was similar to that of the nanocapsules prepared with the non-radioactive drug, indicating that the use of a radiolabeled drug did not modify the physicochemical characteristics of the nanocapsules. The non-chitosan-coated nanocapsules prepared with pluronic F- $68^{\circ}$ showed a droplet a size of $230 \pm 3.2 \mathrm{~nm}$, a Pdl of 0.18 and a zeta potential of $-21.1 \pm 0.06 \mathrm{mV}$. These characteristics were consistent to the nanocapsules synthesized in the previous works.

\subsection{Stability of the nanocapsules upon storage}

The storage of nanocapsule dispersions was investigated at 4 and $25^{\circ} \mathrm{C}$. Also, the stability of the nanocapsules, as a dried powder, was evaluated after elimination of the water from the dispersions. The particle size and the zeta potential were the parameters of evaluation of the nanocapsules dispersions stability over a period of 6 months. Figure 4 showed that nanocapsules dispersion stored at $4{ }^{\circ} \mathrm{C}$ maintained the initial properties and no aggregation was observed $(p>0.05)$. The nanocapsules' dispersion stored at $25^{\circ} \mathrm{C}$ remained stable over 3 months with only a slight variation of their mean diameter and zeta potential by 5 and $7 \%$, respectively. After 6 months, this sample presented a statistic significant increase of the particle size by $19 \%$ while the zeta potential decreased by $20 \%(p<0.05)$. These effects were probably caused by the aggregation process, which is more pronounced at elevated temperatures. These results were consistent to Lemoine et al. and Coffin et al. in which minor changes in the nanoparticles diameter size was observed when stored at 4 or $5{ }^{\circ} \mathrm{C}$ in contrast to higher temperatures ( 25 or $37^{\circ} \mathrm{C}$ ) (Coffin and McGinity, 1992; Lemoine et al., 1996). Storage of formulations under a dried form is generally preferable to avoid instability processes. On this study this was also of utmost importance because of the objective to further produce an oral tablet. However, one major problem with nanoparticles formulations is that they often aggregate during the drying process. Therefore, at the end, the redispersed dried nanoparticles after water addition did not keep the same diameter size characteristics of the original formulation. Indeed, besides irreversible aggregation, nanocapsules can be destroyed by the drying process (Tewa-Tagne et al., 2007). In this work the nanocapsules were dried and characterized by DLS and TEM after redispersion to reconstitute the parent dispersion. Particle size (513.5 $\pm 5.4 \mathrm{~nm}$ with Pdl of 0.19), zeta potential (+37.2 $\pm 0.2 \mathrm{mV})$ and morphology of the nanocapsules appeared to be similar to the initial dispersion after drying and reconstitution in water (Figure 2 and $3 \mathrm{C}$ ). Comparing the size and Pdl of the two nanocapsule dispersions, the difference was not statistically significant $(p>0.05)$. The nanostructure as observed by TEM was also well-preserved demonstrating that paclitaxel loaded copaiba oil-chitosanpoly(isobutyl cyanoacrylate) core-shell nanocapsules can be dried without losing their physicochemical characteristics and morphology. 


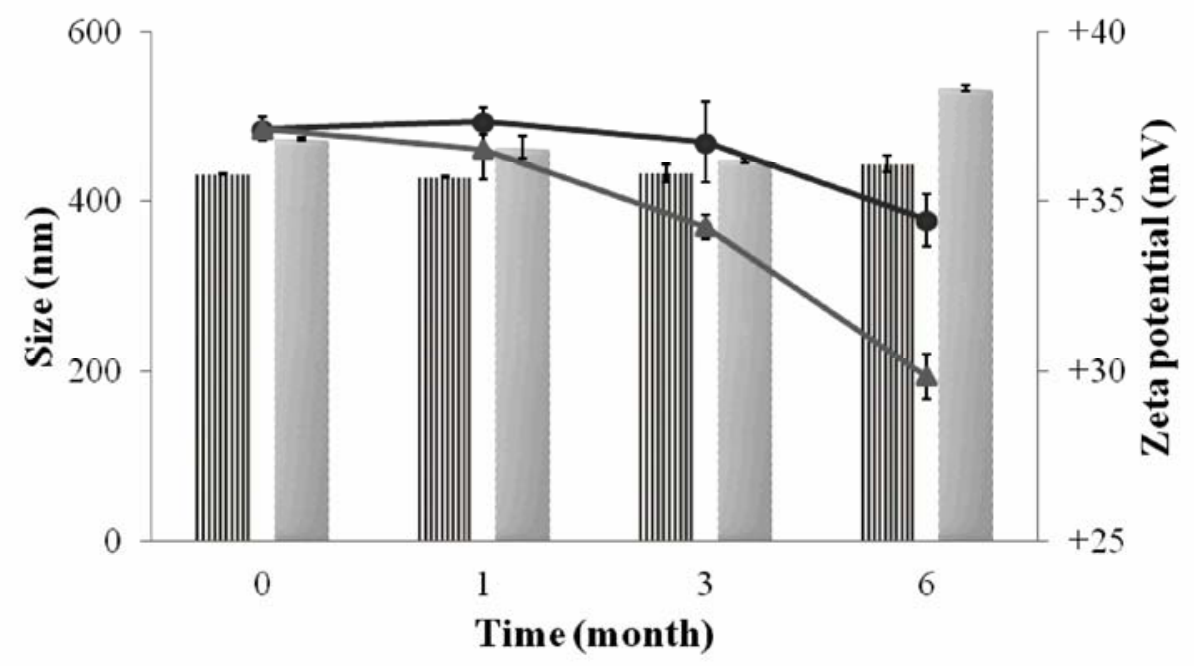

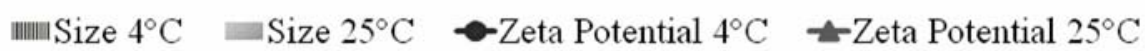

Figure 4- Particle size $(n=3)$ and zeta potential $(n=3)$ of paclitaxel loaded copaiba oilchitosan-poly(isobutyl cyanoacrylate) core-shell nanocapsules stored at $4{ }^{\circ} \mathrm{C}$ and $25^{\circ} \mathrm{C}$ over a period of 6 months.

3.4 Stability of the paclitaxel loaded copaiba oil-chitosan-poly(isobutyl cyanocrylate) core-shell nanocapsules in simulated gastrointestinal fluids

The diameter size and the fluorescent image of the labeled nanocapsules were followed over a period of 120 minutes of incubation in simulated gastric and intestinal media (Figure 5 and 6).

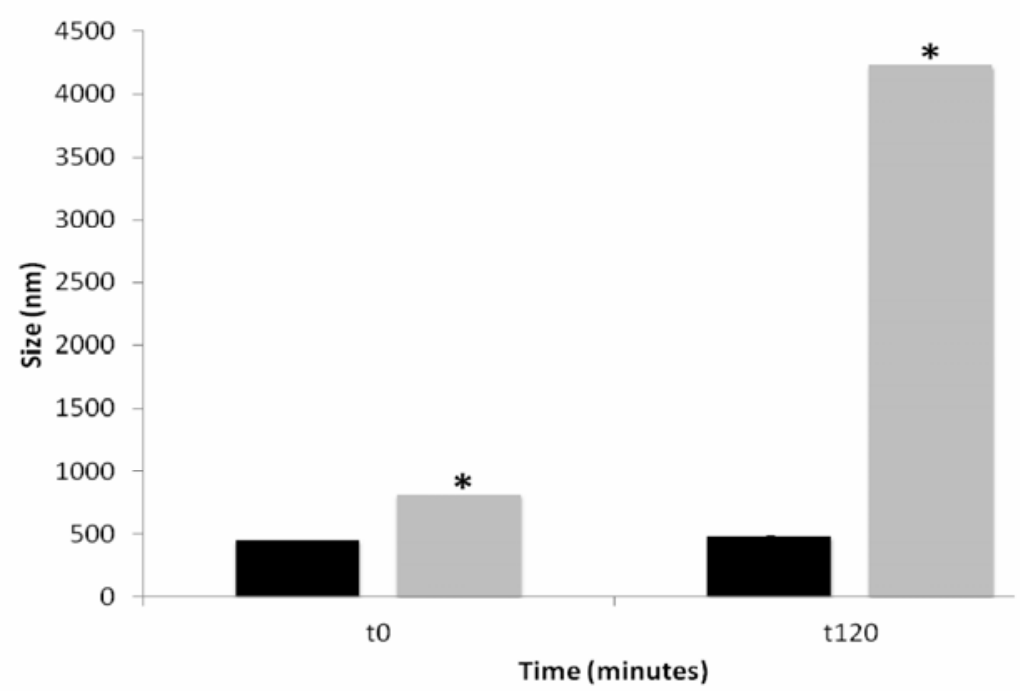

Figure 5- Diameter size $(n=3)$ of polyFluor 570 labeled nanocapsules in different media simulating the $\mathrm{pH}$ environment in the gastrointestinal tract after two hours of incubation. Dark bars $=$ Simulated gastric fluid, light bar $=$ Simulated intestinal fluid, ${ }^{*}=p<0.05$. 


\section{Nanocapsules time (min)}

T0

T30

T60

T120

Water (control)

\section{Gastric Fluid (pH 1.2) Intestinal Fluid (pH 6.8)}
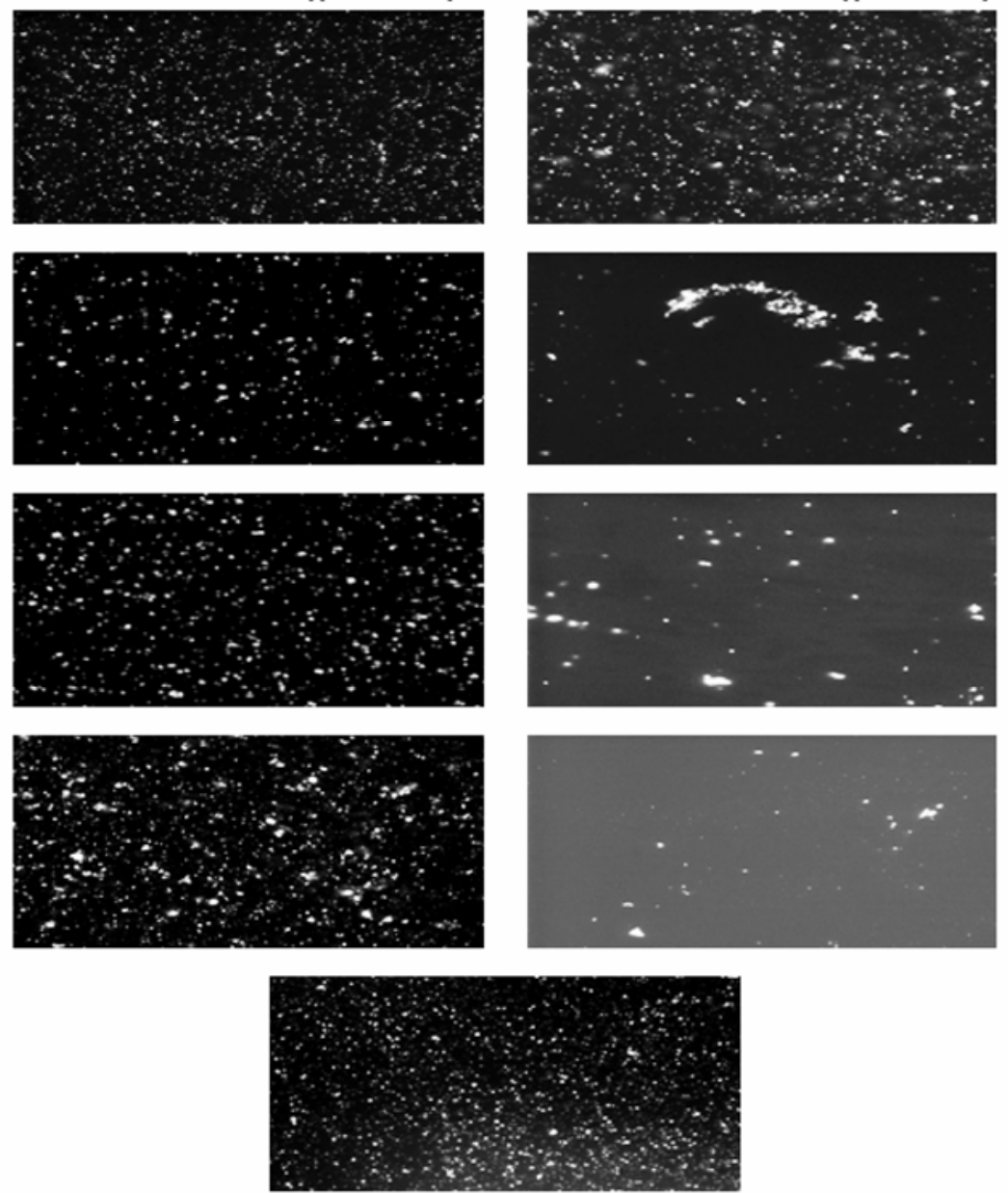

Figure 6- Polyfluor 570 labeled nanocapsules stability in reconstituted gastric and intestinal fluids over 120 minutes.

In the reconstituted gastric medium, no important increase of the size occurred after 120 minutes of incubation ( $p>0.05)$. The fluorescent microscopy revealed that the fluorescence remains confined within the nanocapsules for at least 1 hour and the release of fluorescence clearly started after 120 minutes of incubation in the gastric medium. This indicated that the nanocapsules were quite stable in the gastric environment while they start to leak after 120 minutes in this medium. Although the nanocapsules became leaky, no significant aggregation was observed. At strong acid pH, chitosan, having a pKa of 6.3 , is highly protonated, hence, positively charged, which can explain why the nanocapsules remained well dispersed (Meng et al., 2010).

During the first 30 min of incubation in the intestinal medium, the nanocapsules dispersion showed a dramatic increase on the diameter size, corresponding to five times more, and a PdI of 0.64. Moreover, the fluorescent microscopy revealed a marked fluorescent background. Aggregation of the fluorescent nanocapsules, only shown at 120 minutes, suggests that this system were leaky and already started to degrade along the time. The DLS results revealed the same pattern of degradation. In the intestinal medium, the chitosan protonation is low, hence, the nanocapsules charge. Therefore, nanocapsules may tend to aggregate because the electrostatic forces contributing to the colloidal stability may be reduced. The enzymatic environment is also greatly favorable to the loss of stability of the poly(isobutyl cyanoacrylate) 
nanocapsules. Esterases can degrade the polymer causing leakage as well as swelling of the polymer envelope (Banerjee et al., 2014). The present observations were very consistent with those reported by Aboubakar et al. on the insulin nanocapsules evaluation (Aboubakar et al., 2000).

\subsection{Mucoadhesion studies}

The potential use of small mucoadhesive polymer particle formulations lies in possible prolongation of the residence time near the absorption sites of the drug either through nonspecific (van der Waals and/or hydrophobic interactions) or specific interactions between components of the particles and the mucus. In general, increases of the bioavailability of the loaded drug were, then, obtained (Moghaddam et al., 2009; Ponchel and Irache, 1998). Mucus, which is composed of highly hydrated glycoprotein called mucins, covers the mucosa, forming a continuous adherent blanket on the surface of the epithelium. While adhering to the mucosa, nanocapsules would have to interact with mucins. There are several suitable methods to investigate mucoadhesion of nanocapsules on the gut mucosa (Laffleur and Schnurch, 2013; Neves et al., 2011). For instance, the method of evaluating aggregation of nanocapsules in presence of mucin was selected as a convenient in-vitro test. Adhesion of nanocapsules was also evaluated, directly, on the rat intestinal mucosa. Results from the aggregation of unloaded and loaded-paclitaxel nanocapsules, coated with chitosan, with mucin were presented in Figure 7. Such results were compared to the ones obtained with nanocapsules composed of poly(isobutyl cyanoacrylate) copaiba oil without the coating of chitosan. These nanocapsules were stabilized by pluronic $\mathrm{F}-68^{\circ}$, including PEG chains that are also know to display mucoadhesive properties (Gratieri et al., 2010; Jones et al., 2009).

Different profiles of mucin-nanocapsules aggregation were obtained comparing the different types of nanocapsules. At concentrations bellow $2.5 \mathrm{mg} \cdot \mathrm{mL}^{-1}$ the loaded and unloaded paclitaxel nanocapsules coated with chitosan showed a remarked difference. Above this concentration, the nanocapsules showed a maximum of aggregation in the presence of mucins, which were acknowledged by the plateau observed on the curves. The occurrence of the plateau indicated the occurrence of a saturation phenomenon. This was consistent considering that interactions between the nanocapsules and the mucins consisted of chemical bonds between the positively charged amino groups of the chitosan and the negatively charged sialic acid residues of the mucus glycoproteins at concentrations above $2.5 \mathrm{mg} \cdot \mathrm{mL}^{-1}$ in the nanocapsules (Rossi et al., 2000). As it can be observed in Figure 7, the presence of pluronic $\mathrm{F}-68^{\circ}$ on the nanocapsules surface did not induce aggregation of the mucin over the range of the studied concentrations. In contrast with the nanocapsules coated with chitosan, the nanocapsules coated with pluronic F- $68^{\circ}$ did not interacted with mucins suggesting that the addition of chitosan on the nanocapsules surface was responsible by their mucoadhesion. Mucoadhesion of the nanocapsules was also evaluated on freshly excised rat intestinal mucosa while the tissue was mounted in an Ussing Chamber. The nanocapsules were loaded with [3H]paclitaxel. Thus, only mucoadhesion of the radiolabeled nanocapsules could be evaluated by this method. The used nanocapsules concentration was the same as in the plateau observed in the mucin aggregation test described above. After incubation of the nanocapsules with the mucosa in the Ussing Chambers, $9 \pm 1.1 \%$ of the initial amount of the radioactivity introduced by the nanocapsules was associated to the mucosa after two hours. This corresponded to a deposition and strong attachment of $6.2 \times 10^{9}$ nanocapsules per square centimeter of the intestinal mucosa, or to $3.4 \mathrm{~g}$ of nanocapsules per square meter. 


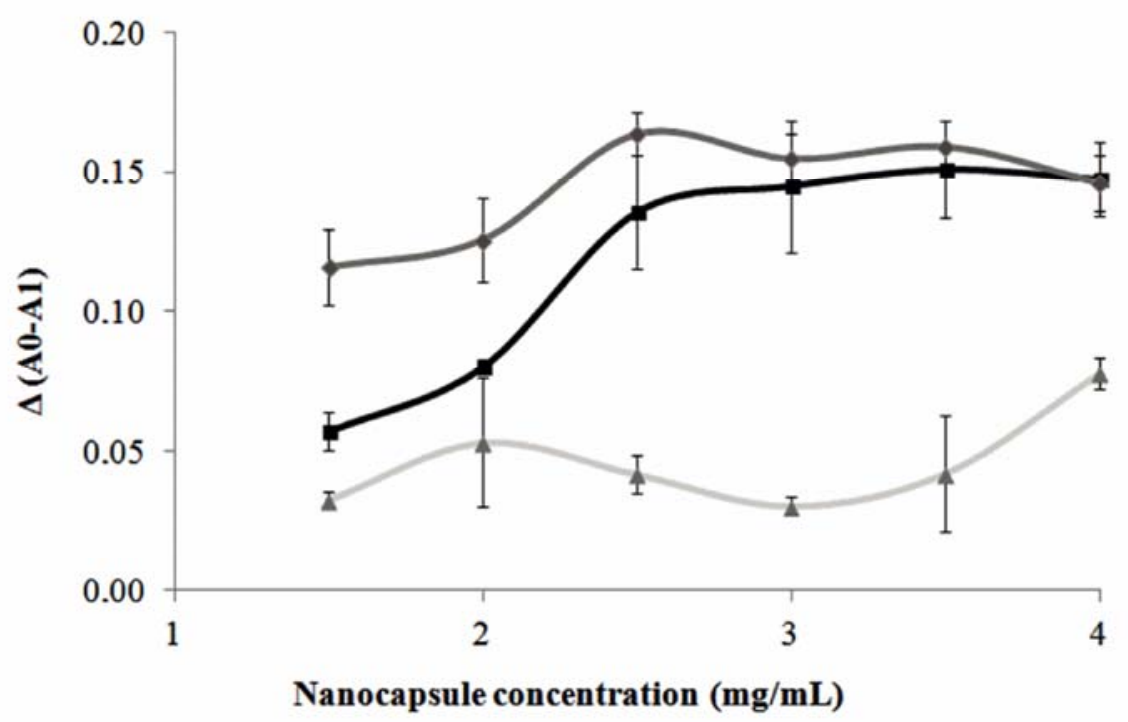

Figure 7-In-vitro mucin aggregation test $(n=3)$ to evaluate mucoadhesive properties of the nanocapsules. NCC: Copaiba oil-loaded chitosan-poly (isobutyl cyanoacrylate) core-shell nanocapsules (- - $)$; NCC PTX: Paclitaxel loaded NCC ( $-(-)$ ); NC-Plu: Copaiba oil-loaded pluronic-poly (isobutyl cyanoacrylate) nanocapsules $(-\Delta-$ ).

In the literature, Bravo-Osuna et al. (2007) have observed that the presence of chitosan on the poly(isobutyl cyanoacrylate) nanoparticles surface increased dramatically the mucoadhesive behavior of the nanoparticles thanks to the formation of hydrogen and ionic bonds between the positively charged amino groups of the polysaccharide and the negatively charged sialic acid residues of mucin glycoproteins (Bravo-Osuna et al., 2007b). The authors reported that the amount of attached chitosan $20 \mathrm{kDa}$-coated nanoparticles per square meter of intestinal mucosa of rat was $2 \mathrm{mg} \cdot \mathrm{mL}^{-1}$, corresponding to a nanoparticles adhesive interaction of about $1.5 \mathrm{~g} / \mathrm{m}^{2}$. Thus, comparing the studies developed in this work with the ones from Bravo-Osuna et al., an increase by 2 -fold in the nanocapsules adhesive interaction with the mucosa was observed. Such results are in complete agreement to those reported by Moghaddam et al. (2009), who have found approximately the same amount of nanoparticles attached to the intestinal mucosa of rats while investigating the mucoadhesion of chitosan 20kDa-pHEMA nanoparticles (Moghaddam et al., 2009).

In the clinic, paclitaxel dose is commonly prescribed at $175 \mathrm{mg} / \mathrm{m}^{2}$ and administered intravenously over a period of 3-24 hours (van den Bongard et al., 2004). Sacco et al. (2010) observed an overall mean body surface area of $1.79 \mathrm{~m}^{2}(95 \% \mathrm{Cl} 1.78-1.80)$ for patients receiving chemotherapy for treat head and neck, ovarian, lung, upper $\mathrm{Gl} /$ pancreas, breast or colorectal cancers (Sacco et al., 2010). Thus, to obtain an ideal anticancer therapy, a dose of $313.3 \mathrm{mg}$ of paclitaxel would be required. Moreover, the paclitaxel loaded copaiba oilpoly(isobutyl cyanoacrylate) nanocapsules coated with chitosan developed in this work show an important impact as a new anticancer therapy by oral route. Indeed, taking into account the ideal therapy, $18.6 \mathrm{~g}$ of these nanocapsules would be required. Although the amount administered of nanocapsules appears to be high for oral route, other factors such as the synergic effect of copaiba oil in the cancer therapy and the changes on the bioavailability induced by the modified release of this system have not been considered in order to reduce the traditional dose. Therefore, the therapeutic system here proposed could present a higher convenience for patient and better compliance to treatment due to the non-invasive administration of the cancer drugs. 


\section{Conclusions}

New poly(isobutyl cyanoacrylate) based nanocapsules were synthesized by interfacial polymerization having a surface coated with chitosan. Paclitaxel could be incorporated in the nanocapsules, which cavity was filled out with copaiba oil, while chitosan conferred interesting mucoadhesive properties to the new formulation. Properties of the nanocapsules agreed well with those expected for a formulation designed to enhance oral bioavailability of the associated drug. Taking together, the results from the present work are encouraging to pursue the development of the chitosan-coated nanocapsules for oral delivery of paclitaxel as a new treatment for cancer with possible synergetic anticancer effect with therapeutically active components found in the copaiba oil.

Acknowledgements: The authors would like to thank the financial support from the "Coordenação de Aperfeiçoamento de Pessoal de Nível Superior- CAPES" through the COFECUB 721/11 project for Xavier-Jr., F.H. fellowship. The authors are also grateful to Imagif Cell Biology Unit of the Gif campus, Conseil Général de l'Essonne by images analyses (www.imagif.cnrs.fr) for the access of the TEM facility.

Author Contributions: Author FHXJ and CV designed the experiments, analyzed the data and wrote the manuscript. Author CG provided assistance on HPLC dosages. HC made a significant contribution for radioactivity assays. Corresponding author ESTE was responsible to write-review and edit, supervision, project administration and funding acquisition.

Conflicts of Interest: The authors declare no conflict of interest.

\section{References}

Aboubakar, M., Couvreur, P., Pinto-Alphandary, H., Gouritin, B., Lacour, B., Farinotti, R., Puisieux, F., Vauthier, C., 2000. Insulin-loaded nanocapsules for oral administration: In vitro and in vivo investigation. Drug Dev Res 49, 109-117.

Aboubakar, M., Puisieux, F., Couvreur, P., Vauthier, C., 1999. Physico-chemical characterization of insulin-loaded poly(isobutylcyanoacrylate) nanocapsules obtained by interfacial polymerization. Int J Pharm 183, 63-66.

Agueros, M., Zabaleta, V., Espuelas, S., Campanero, M.A., Irache, J.M., 2010. Increased oral bioavailability of paclitaxel by its encapsulation through complex formation with cyclodextrins in poly(anhydride) nanoparticles. J Control Release 145, 2-8.

Al Khouri Fallouh, N., Roblot-Treupel, L., Fessi, H., Devissaguet, J.P., Puisieux, F., 1986. Development of a new process for the manufacture of polyisobutylcyanoacrylate nanocapsules. Inter J Pharm 28, 125-132.

Anand, N., Kanwar, R.K., Sehgal, R., Kanwar, J.R., 2016. Antiparasitic and immunomodulatory potential of oral nanocapsules encapsulated lactoferrin protein against Plasmodium berghei. Nanomedicine 11, 47-62.

Anand, N., Sehgal, R., Kanwar, R.K., Dubey, M.L., Vasishta, R.K., Kanwar, J.R., 2015. Oral administration of encapsulated bovine lactoferrin protein nanocapsules against intracellular parasite Toxoplasma gondii. Int J Nanomedicine 10, 6355-6369. 
Banerjee, A., Chatterjee, K., Madras, G., 2014. Enzymatic degradation of polymers: a brief review. Mater Sci Technol 30, 567-573.

Bathool, A., Vishakante, G.D., Khan, M.S., Shivakumar, H.G., 2012. Development and characterization of atorvastatin calcium loaded chitosan nanoparticles for sustain drug delivery Adv Mat Lett 3, 466-470.

Bertholon, I., Ponchel, G., Labarre, D., Couvreur, P., Vauthier, C., 2006. Bioadhesive properties of poly(alkylcyanoacrylate) nanoparticles coated with polysaccharide. J Nanosci Nanotechnol 6, 3102-3109.

Bravo-Osuna, I., Ponchel, G., Vauthier, C., 2007a. Tuning of shell and core characteristics of chitosan-decorated acrylic nanoparticles. Eur J Pharm Sci 30, 143-154.

Bravo-Osuna, I., Vauthier, C., Farabollini, A., Palmieri, G.F., Ponchel, G., 2007b. Mucoadhesion mechanism of chitosan and thiolated chitosan-poly(isobutyl cyanoacrylate) core-shell nanoparticles. Biomaterials 28, 2233-2243.

Buzea, C., Pacheco, II, Robbie, K., 2007. Nanomaterials and nanoparticles: sources and toxicity. Biointerphases 2, MR17-71.

Calleja, P., Espuelas, S., Corrales, L., Pio, R., Irache, J.M., 2014. Pharmacokinetics and antitumor efficacy of paclitaxel-cyclodextrin complexes loaded in mucus-penetrating nanoparticles for oral administration. Nanomedicine 9, 2109-2121.

Calleja, P., Espuelas, S., Vauthier, C., Ponchel, G., Irache, J.M., 2015. controlled release, intestinal transport, and oral bioavailablity of paclitaxel can be considerably increased using suitably tailored pegylated poly(anhydride) nanoparticles. J Pharm Sci 104, 2877-2886.

Cho, W.S., Thielbeer, F., Duffin, R., Johansson, E.M., Megson, I.L., MacNee, W., Bradley, M., Donaldson, K., 2014. Surface functionalization affects the zeta potential, coronal stability and membranolytic activity of polymeric nanoparticles. Nanotoxicology 8, 202-211.

Coffin, M.D., McGinity, J.W., 1992. Biodegradable pseudolatexes: the chemical stability of poly(D,L-lactide) and poly(epsilon-caprolactone) nanoparticles in aqueous media. Pharm Res 9, 200-205.

Convention, U.S.P., 2011. U.S. Pharmacopeia National Formulary United States Pharmacopeial: Pck Sup edition.

Danhier, F., Lecouturier, N., Vroman, B., Jerome, C., Marchand-Brynaert, J., Feron, O., Preat, V., 2009. Paclitaxel-loaded PEGylated PLGA-based nanoparticles: in vitro and in vivo evaluation. J Control Release 133, 11-17.

Danhier, F., Ucakar, B., Magotteaux, N., Brewster, M.E., Preat, V., 2010. Active and passive tumor targeting of a novel poorly soluble cyclin dependent kinase inhibitor, JNJ-7706621. Int J Pharm 392, 20-28.

Gratieri, T., Gelfuso, G.M., Rocha, E.M., Sarmento, V.H., Freitas, O., Lopez, R.F., 2010. A poloxamer/chitosan in situ forming gel with prolonged retention time for ocular delivery. Eur J Pharm Biopharm 75, 186-193.

Huang, C.Y., Chen, C.M., Lee, Y.D., 2007. Synthesis of high loading and encapsulation efficient paclitaxel-loaded poly(n-butyl cyanoacrylate) nanoparticles via miniemulsion. Int J Pharm 338, 267-275. 
Jabr-Milane, L.S., van Vlerken, L.E., Yadav, S., Amiji, M.M., 2008. Multi-functional nanocarriers to overcome tumor drug resistance. Cancer Treat Rev 34, 592-602.

Jones, D.S., Bruschi, M.L., Freitas, O., Gremiao, M.P., Lara, E.H., Andrews, G.P., 2009. Rheological, mechanical and mucoadhesive properties of thermoresponsive, bioadhesive binary mixtures composed of poloxamer 407 and carbopol 974P designed as platforms for implantable drug delivery systems for use in the oral cavity. Int J Pharm 372, 49-58.

Laffleur, F., Schnurch, A.B., 2013. Strategies for improving mucosal drug delivery. Nanomedicine 8, 2061-2075.

Lee, W.C., Kopetz, S., Wistuba, I.I., Zhang, J., 2017. Metastasis of cancer: when and how? Ann Oncol 28, 2045-2047.

Lemoine, D., Francois, C., Kedzierewicz, F., Preat, V., Hoffman, M., Maincent, P., 1996. Stability study of nanoparticles of poly(epsilon-caprolactone), poly(D,L-lactide) and poly(D,L-lactide-coglycolide). Biomaterials 17, 2191-2197.

Mazzaferro, S., Bouchemal, K., Ponchel, G., 2013. Oral delivery of anticancer drugs III: formulation using drug delivery systems. Drug Discov Today 18, 99-104.

McConville, J.T., 2017. Current developments in oral drug administration. Drug Dev Ind Pharm 43, 699.

Mendes, A.C., Moreno, J.S., Hanif, M., T, E.L.D., Chen, M., Chronakis, I.S., 2018. Morphological, mechanical and mucoadhesive properties of electrospun chitosan/phospholipid hybrid nanofibers. Int J Mol Sci 19.

Meng, X., Tian, F., Yang, J., He, C.N., Xing, N., Li, F., 2010. Chitosan and alginate polyelectrolyte complex membranes and their properties for wound dressing application. J Mater Sci Mater Med 21, 1751-1759.

Moghaddam, F.A., Atyabi, F., Dinarvand, R., 2009. Preparation and in vitro evaluation of mucoadhesion and permeation enhancement of thiolated chitosan-pHEMA core-shell nanoparticles. Nanomedicine 5, 208-215.

Mohammadpour Dounighi, N., Eskandari, R., Avadi, M., Zolfagharian, H., Mir Mohammad Sadeghi, A., Rezayat, M., 2012. Preparation and in vitro characterization of chitosan nanoparticles containing Mesobuthus eupeus scorpion venom as an antigen delivery system. $J$ Venom Anim Toxins Incl Trop Dis 18, 44-52.

Neves, J., Bahia, M.F., Amiji, M.M., Sarmento, B., 2011. Mucoadhesive nanomedicines: characterization and modulation of mucoadhesion at the nanoscale. Expert Opin Drug Deliv 8, 1085-1104.

Peltier, S., Oger, J.M., Lagarce, F., Couet, W., Benoit, J.P., 2006. Enhanced oral paclitaxel bioavailability after administration of paclitaxel-loaded lipid nanocapsules. Pharm Res 23, 1243-1250.

Ponchel, G., Irache, J., 1998. Specific and non-specific bioadhesive particulate systems for oral delivery to the gastrointestinal tract. Adv Drug Deliv Rev 34, 191-219.

Rivkin, I., Cohen, K., Koffler, J., Melikhov, D., Peer, D., Margalit, R., 2010. Paclitaxel-clusters coated with hyaluronan as selective tumor-targeted nanovectors. Biomater 31, 7106-7114. 
Roger, E., Lagarce, F., Garcion, E., Benoit, J.P., 2010. Reciprocal competition between lipid nanocapsules and P-gp for paclitaxel transport across Caco-2 cells. Eur J Pharm Sci 40, 422-429.

Rossi, S., Ferrari, F., Bonferoni, M.C., Caramella, C., 2000. Characterization of chitosan hydrochloride-mucin interaction by means of viscosimetric and turbidimetric measurements. Eur J Pharm Sci 10, 251-257.

Sacco, J.J., Botten, J., Macbeth, F., Bagust, A., Clark, P., 2010. The average body surface area of adult cancer patients in the UK: a multicentre retrospective study. PLoS One 5, e8933.

Shamekhi, F., Tamjid, E., Khajeh, K., 2018. Development of chitosan coated calcium-alginate nanocapsules for oral delivery of liraglutide to diabetic patients. Int J Biol Macromol 120, 460467.

Tewa-Tagne, P., Briancon, S., Fessi, H., 2007. Preparation of redispersible dry nanocapsules by means of spray-drying: development and characterisation. Eur J Pharm Sci 30, 124-135.

Thwala, L.N., Delgado, D.P., Leone, K., Marigo, I., Bennetti, F., Chenlo, M., Alvarez, C.V., Tovar, S., Dieguez, C., Csaba, N.S., Alonso, M.J., 2018. Protamine nanocapsules as carriers for oral peptide delivery. J Control Release.

van den Bongard, H.J., Kemper, E.M., van Tellingen, O., Rosing, H., Mathot, R.A., Schellens, J.H., Beijnen, J.H., 2004. Development and validation of a method to determine the unbound paclitaxel fraction in human plasma. Anal Biochem 324, 11-15.

Vauthier, C., Bouchemal, K., 2009. Methods for the preparation and manufacture of polymeric nanoparticles. Pharm Res 26, 1025-1058.

Wang, Q., Li, C., Ren, T., Chen, S., Ye, X., Guo, H., He, H., Zhang, Y., Yin, T., Liang, X.J., Tang, X., 2017. Poly(vinyl methyl ether/maleic anhydride)-doped PEG-PLA nanoparticles for oral paclitaxel delivery to improve bioadhesive efficiency. Mol Pharm 14, 3598-3608.

Wani, M.C., Taylor, H.L., Wall, M.E., Coggon, P., McPhail, A.T., 1971. Plant antitumor agents. VI. The isolation and structure of taxol, a novel antileukemic and antitumor agent from Taxus brevifolia. J Am Chem Soc 93, 2325-2327.

Wei, W., Lv, P., Ma, G., 2015. Oral delivery of protein and anticancer drugs by uniform-sized chitosan micro/nanoparticles with autofluorescent property. J Control Release 213, e111.

Xavier-Junior, F.H., Egito, E.S.T., Morais, A.R.V., Alencar, E.N., Maciuk, A., Vauthier, C., 2018. Experimental design approach applied to the development of chitosan coated poly(isobutylcyanoacrylate) nanocapsules encapsulating copaiba oil. Colloids Surf A Physicochem Eng Asp 536, 251-258.

Xavier-Junior, F.H., Gueutin, C., Morais, A.R.V., Alencar, E.N., Egito, E.S.T., Vauthier, C., 2016. HPLC method for the dosage of paclitaxel in copaiba oil: Development, validation, application to the determination of the solubility and partition coefficients. Chromatographia 79, 405-412.

Zabaleta, V., Calleja, P., Espuelas, S., Corrales, L., Pio, R., Agueros, M., Irache, J.M., 2013. Mucopenetrating nanoparticles: vehicles for the oral administration of paclitaxel. Ann Pharm Fr 71, 109-118. 